\title{
AWARDS
}

\section{Optics underpins astronomy Nobel}

Exquisite optical engineering has once again helped astronomy win a Nobel Prize in Physics. One half of the 2019 prize has been awarded to Michel Mayor and Didier Queloz for the "discovery of an exoplanet orbiting a solar-type star" (https:// go.nature.com/2NURrW2).

In October 1995, in a landmark paper in Nature ${ }^{1}$, the duo reported a Jupitermass companion around the star 51 Pegasi in the constellation of Pegasus. Notably, this observation was made by measuring tiny Doppler frequency shifts in the spectral absorption lines of a star that occur when the presence of an orbiting planet slightly perturbs the star's motion as seen from Earth. Such measurements, usually quoted in terms of variation in the star's radial velocity, are very small and thus highly demanding. In the 1990s, Mayor and Queloz from the University of Geneva in Switzerland decided to build a custom-designed, highly sensitive echelle spectrograph in order to improve the precision of measurements and expand observations to dimmer stars.

The resulting ELODIE instrument was installed at the Haute-Provence Observatory in France (pictured) and was able to make radial velocity measurements with an accuracy of $13 \mathrm{~m} \mathrm{~s}^{-1}$ for stars of up to magnitude 9 in brightness in an exposure time of within $30 \mathrm{~min}$. ELODIE was novel in that instead of using an absorption cell and a slit to measure spectral shifts in stellar absorption lines it used a dual optical fibre delivery system, an echelle grating and a charge-coupled device (CCD) camera (https://go.nature.com/2NqzPCx). One fibre delivered the starlight and the other reference light from a thorium-argon calibration lamp, while the echelle grating performed high-order diffraction and the CCD camera digitally recorded the resulting spectrum between 390 to $680 \mathrm{~nm}$. The result was a spectrograph with a resolving power of 42,000 , and, importantly, the radial velocity accuracy of just $13 \mathrm{~m} \mathrm{~s}^{-1}$ was precise enough to detect the $59 \mathrm{~m} \mathrm{~s}^{-1}$ variation associated with the 51 Pegasi exoplanet. The exoplanet was determined to have a mass of about half of Jupiter and an orbital period of about 4 days.

The observation of this first exoplanet around a main-sequence star ignited

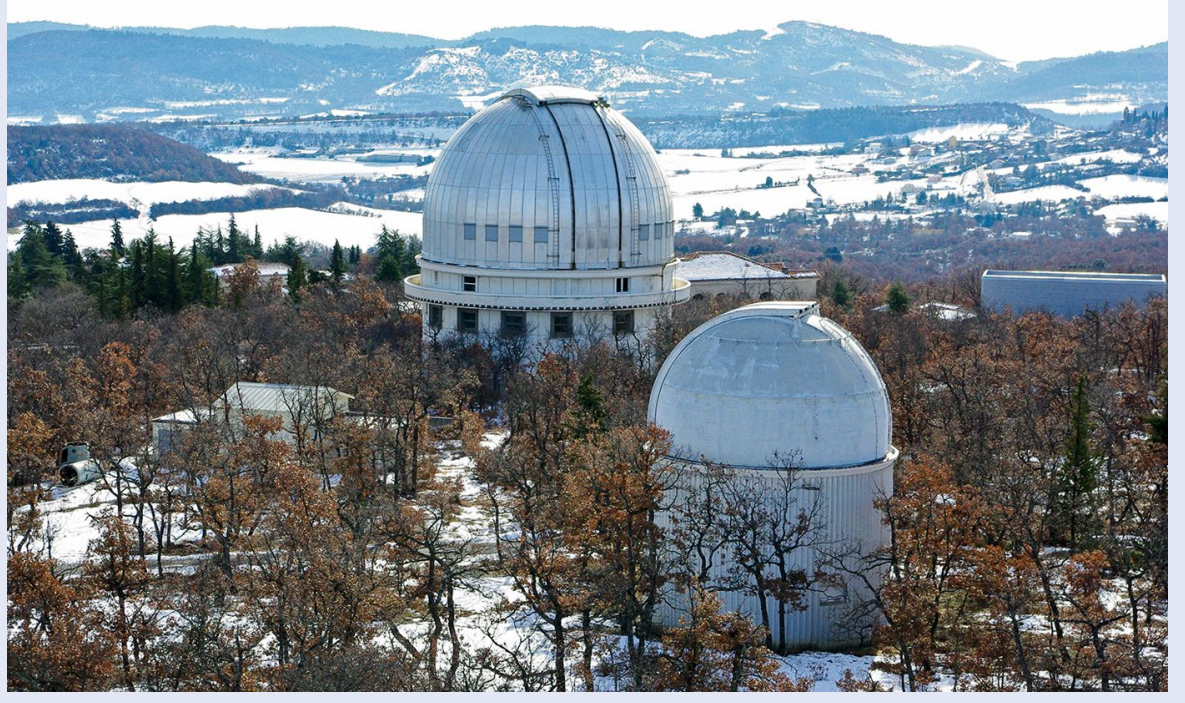

Credit: (c) Thierry Gauquelin / CNRS Photothèque

the field of exoplanet detection that subsequently exploded into activity. Today, more than 4,000 exoplanets have been reported. In recent times, the preferred search technique is the use of transit observations, where an exoplanet temporarily blocks some of the light from a star as it moves across the star during its orbit. The result is a temporary dip in the strength of the signal detected on Earth.

Meanwhile, the development of optical frequency combs - a highly precise spectral ruler of light made of a large number of equally spaced narrow spectral lines - is fuelling further improvements in the accuracy of radial velocity measurements. In the January 2019 issue of Nature Photonics, two research groups independently reported how the use of combs as a reference and calibration source can enable radial velocity measurements on the sub-metre-per-second scale ${ }^{2,3}$. As for ELODIE, it was decommissioned in August 2006 after 12 years of observations and its successor at the Haute-Provence Observatory is another echelle spectrograph with superior characteristics named SOPHIE (https://go.nature.com/2K1ky90).

This year's Nobel comes just 24 months after an earlier feat in photonics helped astronomy receive another Nobel for the long-awaited detection of gravitational waves. In October 2017, Rainer Weiss, Barry Barish and Kip Thorne were recipients of the Nobel Prize in Physics for the detection of gravitational waves in 2015 by the advanced Laser Interferometer Gravitational-Wave Observatory (aLIGO; https://go.nature.com/2NqRZ7g) in the US (https://go.nature.com/2CowduH). aLIGO is the largest and most sensitive optical interferometer that has ever been built. The design is based on a Michelson interferometer with 4-km-long arms held under ultrahigh vaccum and can measure a change in arm length of the order of $10^{-19} \mathrm{~m}$ as a gravitational wave passes through the instrument ${ }^{4}$.

We eagerly await to see what future breakthroughs in astronomy will result from further developments in the capabilities of photonic instrumentation.

Oliver Graydon

Published online: 22 November 2019

https://doi.org/10.1038/s41566-019-0565-5

\section{References}

1. Mayor, M. \& Queloz, D. Nature 378, 355-359 (1995).

2. Obrzud, E. et al. Nat. Photon. 13, 31-35 (2019).

3. Suh, M.-G. et al. Nat. Photon. 13, 25-30 (2019).

4. Nat. Photon. 11, 677 (2017). 